\title{
Minimum Information Necessary for Quantitative Real-Time PCR Experiments
}

\author{
Gemma Johnson, Afif Abdel Nour, Tania Nolan, Jim Huggett, \\ and Stephen Bustin
}

\begin{abstract}
The MIQE (minimum information for the publication of quantitative real-time PCR) guidelines were published in 2009 with the twin aims of providing a blueprint for good real-time quantitative polymerase chain reaction (qPCR) assay design and encouraging the comprehensive reporting of qPCR protocols. It had become increasingly clear that variable pre-assay conditions, poor assay design, and incorrect data analysis were leading to the routine publication of data that were often inconsistent, inaccurate, and wrong. The problem was exacerbated by a lack of transparency of reporting, with the details of technical information inadequate for the purpose of assessing the validity of published qPCR data. This had, and continues to have serious implications for basic research, reducing the potential for translating findings into valuable applications and potentially devastating consequences for clinical practice. Today, the rationale underlying the MIQE guidelines has become widely accepted, with more than 2,200 citations by March 2014 and editorials in Nature and related publications acknowledging the enormity of the problem. However, the problem we now face is rather serious: thousands of publications that report suspect data are populating and corrupting the peer-reviewed scientific literature. It will be some time before the many contradictions apparent in every area of the life sciences are corrected.
\end{abstract}

Key words PCR, Reverse transcription, Diagnostics, Gene expression

\section{Introduction}

The MIQE (minimum information for the publication of quantitative real-time PCR) guidelines [1] represent a major milestone in the transformation of the real-time quantitative polymerase chain reaction (qPCR) from a research technique into a reliable "gold standard." A comparison of qPCR with conventional endpoint PCR reveals that qPCR is less prone to contamination, easier to implement, requires less hands-on time, has the potential for high throughput, and can be quantitative. This has allowed it to rapidly displace legacy PCR for many applications, making it into a ubiquitous technique capable of delivering numerous results in minimal 

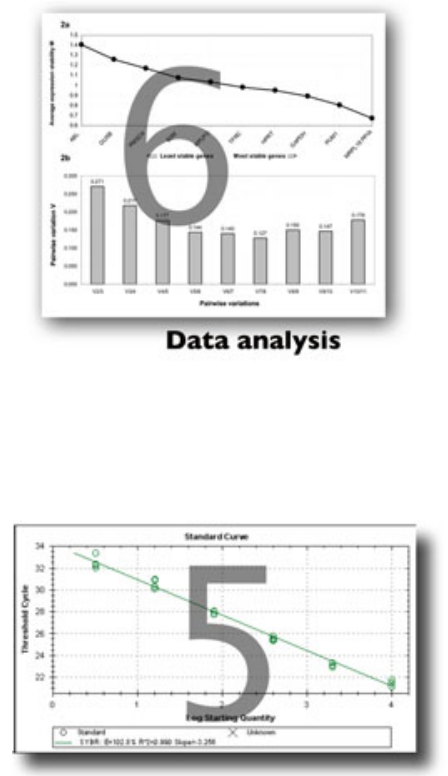

qPCR validation

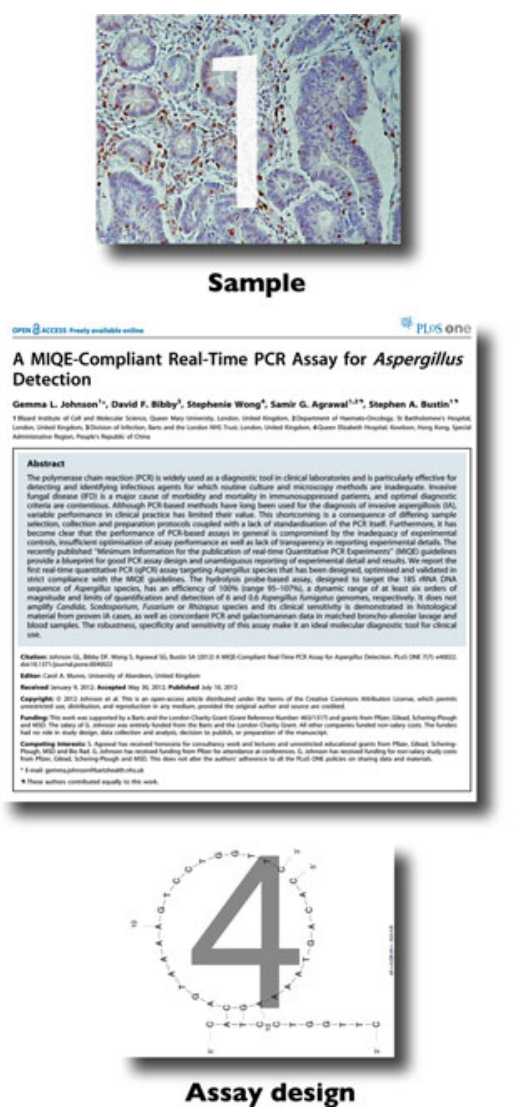

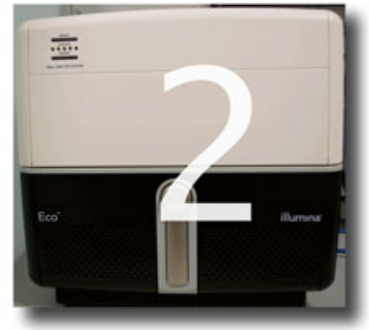

Instrument and reagents

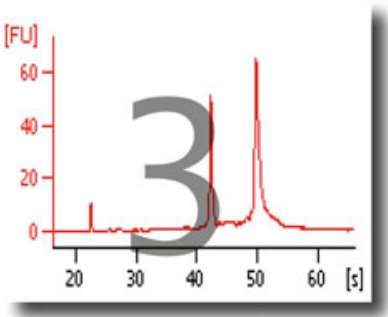

Nucleic acid quality assessment

Fig. 1 A qPCR publication depends on the successful completion of a series of steps, each one of which must be carefully quality controlled to ensure reliable, accurate, and reproducible amplification

time. The simplicity of data acquisition has fostered the impression that qPCR data are robust and reliable, but has failed to impress on researchers that there are numerous critical steps associated with a successful qPCR assay, every one of which needs to be quality controlled for the results to be meaningful (Fig. 1). Unfortunately, it has been clear for some time that the quantity of qPCR data is not matched by an equivalent quality. As a consequence, there are numerous publications reporting contradictory data and results are frequently not reproducible, yet are circulated in the peer-reviewed literature without any obvious criteria to distinguish a genuine result from a technical artifact.

A particular low point came with the revelations concerning the inappropriate use of the reverse transcription (RT)-qPCR in publications associating measles virus with novel gut pathology and autism [2]. A public dissection of published data at the Washington DC autism trial in 1997 revealed a catalogue of inconsistencies, including the use of inappropriate samples, protocols, and analysis methods, ignoring of negative controls that were 
positive and amplification of DNA contaminants. This resulted in a conclusion "confirming" an association between the presence of measles virus and gut pathology in children with developmental disorder. The results were widely used to link the measles, mumps, and Rubella (MMR) vaccine to the occurrence of autism in children and provide a graphical example of the damage an improperly conducted and inadequately published scientific study can do. Other, less egregious examples of the problems that arise from poor experimental practice include the controversy surrounding the lack of association of xenotropic murine leukaemia virus-related virus (XMRV) in prostate cancer [3] and chronic fatigue syndrome [4] and the retraction of a paper describing the migration of mRNA to initiate flowering, which was a "breakthrough of the year" [5].

In response, a growing consensus has been developing around the need to improve the transparency of reporting of relevant experimental detail to include every aspect important to the qPCR assay itself as well as issues relating to pre- and post-assay parameters. Specifically, it became clear that there is a requirement for a set of recommendations that can be used by journal reviewers, who need to be able to evaluate the reliability of the experimental protocols and ensure the inclusion of all essential information in the final publication. Whilst there had been numerous individual papers highlighting the inadequacies, misconceptions, and failures of this important and ubiquitous enabling technology (reviewed in ref. 6), there had been no unifying proposals for a solution to these problems. This need was addressed by the publication of the MIQE guidelines, coauthored by an international group of researchers with a long history of involvement in addressing quality-related issues. For the first time there was a focus that enabled other researchers, journal editors, and non-qPCR expert readers of publications to understand what to look for when evaluating the reliability of conclusions derived from publications utilizing qPCR-based technologies.

There has been a rapid expansion in the number of researchers aware of the existence of these guidelines as well as an increasing number of citations of the original publication in the peer-reviewed literature (> 2,200 by March 2014). There even is an iOS/Android app available for mobile telephones and tablet computers [7]. The final acceptance of the need for guidelines such as these was an editorial in Nature, published in April 2013, which acknowledged that "journals such as this one compound them [the problems] when they fail to exert sufficient scrutiny over the results that they publish" and called for a "checklist" that "focuses on a few experimental and analytical design elements that are crucial for the interpretation of research results but are often reported incompletely" [8]. As a result, Nature and its associated journals no longer have space restrictions on the methods section and even though this conversion by Nature is very late, it is nonetheless welcome. 
It is certainly a long way from the 2010 Nature Medicine report on MIQE, which quoted its editor's attitude as "We would be delighted to embrace the [MIQE] guidelines, but we are not really persuaded that the guidelines are embraced by the community" [9]. It is unfortunate that there was no sign of leadership from the high impact factor journals then, as their support would have accelerated the acceptance of the guidelines.

\section{The Guidelines}

The MIQE guidelines offer a strategy for reproducibility and quality control that allows scientists to cultivate better practices in quantitative PCR experiments [10]. Their fundamental goal is to encourage the publication of transparent and comprehensive technical detail, since this allows a reader to take technical excellence for granted and to focus on the biological relevance of that publication's conclusions. A corollary is that they include all the information required to design, validate, and optimize an assay from scratch and so constitute a blueprint for good assay design. Anyone using MIQE as the basis for developing a qPCR-based assay is virtually guaranteed to achieve that goal and obtain an efficient, specific, and sensitive assay.

MIQE consists of nine sections, with 85 parameters that constitute the minimum information required to allow potential reproduction as well as unambiguous quality assessment of a qPCR-based experiment. These nine sections comprise

- Experimental design

- Sample properties

- Nucleic acid extraction and quality assessment

- Reverse transcription

- Target information

- Primer and probe details

- qPCR protocol optimization and validation details

- Data analysis

The 85 parameters fall into two categories: some are deemed to be essential and are labeled " $E$ " in the published guidelines, because they are indispensable for an adequate description of the qPCR assay. Other components are more peripheral and are labeled "D" (desirable), yet represent an effective foundation for the implementation of best practice protocols. Adherence to these parameters also encourages much-needed standardization, especially important when using qPCR assays for diagnostic applications. Importantly, these parameters are based on common sense 
and current best practice and so are not set in stone and remain open for discussion; indeed, a slightly modified version, labeled MIQE précis encompasses the key MIQE parameters essential for publication in Biomed Central (BMC) journals [11]. Most recently, MIQE-style guidelines for minimum information for publication of quantitative digital PCR experiments (dMIQE) have been published [12].

Possibly the most contentious part of the original MIQE guidelines was the essential requirement for publications to report the sequences of any primers used and the suggestion to also report the sequences of any probes. The rationale behind this is rather straightforward: an experiment cannot be reproduced exactly if the primer sequence, one of the principal reagents, is unavailable. Lack of access to a probe sequence, on the other hand, does not preclude analysis of the specificity, efficiency, and sensitivity of an assay; however, for completeness' sake it is but a small step to take for most researchers. Many commercial qPCR assays are not supplied with the primer/probe sequences, since most vendors consider this commercially sensitive information; usually there are also no details provided on empirical validation of each individual assay. Publications utilizing such assays could not satisfy the original MIQE requirements, placing limits on a universal acceptance of MIQE.

Consequently, an amendment of the original guidelines now requires either primer sequences or a clearly defined amplicon context sequence [13]. This guidance was issued based on the assessment that in the absence of full primer sequence disclosure it is possible to achieve an adequate level of transparency, but only if there is an appropriate level of background information and disclosure of validation results on the qPCR assay. Consequently, if primer sequences are not disclosed, a MIQE-compliant publication should institute the same validation criteria used for assays reporting primer/probe sequences. Specifically, when reporting a precise fold-change for a transcript it remains an essential requirement that the PCR efficiency, analytical sensitivity, and specificity of each individual assay be determined. This information should be verified by the investigator for the actual assay that is being reported using the conditions and personnel in their laboratory and not extrapolated from commercial assays validated by the vendors.

It is worth emphasizing that MIQE proposes minimum guidelines; hence more information can be disclosed, if so desired. For example, MIQE requests information about the specificity, PCR efficiency, $r^{2}$ of calibration curves, linear dynamic range, and $C_{\mathrm{q}}$ variation at the limit of detection. Including the data in a table can fulfill these requirements. However, the addition of individual calibration and melt curves in supplementary material would be far more informative and allow the reader to get a much better feel for the quality of the published data. 


\section{Why the Need for Such Detail?}

At first sight the requirement to list 85 individual criteria appears to be rather onerous. However, every one of the parameters is likely to be encountered and addressed during the routine development, optimization, and validation of a qPCR assay. Hence it is usually simply a matter of recording the results, which can then be tabulated and submitted with the manuscript. The use of the MIQE app also simplifies MIQE compliance, as the analyzed data can be exported with a single click and can then be attached as supplemental data to the article.

The information requested for the reverse transcription step provides a handy example of why the guidelines incorporate such detailed criteria. They list five essential (complete reaction conditions, amount of RNA and reaction volume, priming oligonucleotide if using gene-specific priming and concentration, temperature, and time) and three desirable (manufacturer of reagents and catalogue numbers, $C_{\mathrm{q}} \mathrm{s}$ with and without RT, storage conditions of cDNA) parameters. The reason for this is that RT yields depend on total RNA concentration and RT reaction conditions such as the priming strategy, which affects RT efficiency and is different for different target genes [14]. This is demonstrated in Fig. 2, where the $C_{\mathrm{q}} \mathrm{s}$ of various target mRNAs differ according to whether cDNA synthesis was primed by random hexamers, pentadecamers, oligo-dT, or gene-specific primers. Assay details are shown in Table 1. Since it cannot be predicted how different priming methods affect the RT efficiency of each target, it is essential that a detailed description of the protocol and reagents used to convert RNA into cDNA be provided. Furthermore, reverse transcription yields can vary significantly with the choice of reverse transcriptase and, as with the priming strategy, this variation is gene dependent [15]. This variability is demonstrated in Fig. 3, with the maximum $\Delta C_{\mathrm{q}}$ recorded by different RTs ranging from 4.5 (22-fold) and 7 (128-fold), depending on the target.

Quality control of nucleic acids is another example of the detailed reporting suggestions proffered by the MIQE guidelines. Whilst most researchers are aware of the importance of measuring RNA integrity prior to quantification, many fail to ensure adequate purity of their samples. Purity does not refer to a sample's $A_{260} / A_{280}$ ratio, but rather encompasses the absence of inhibitors of either the RT or the PCR reaction. Inhibition is a well-known yet poorly described phenomenon and we were the first to propose a universal method for inhibition testing that involves the use of a template expressed only in potatoes [16]. The technique, called SPUD, compares the $C_{\mathrm{q}}$ s obtained from the amplification of SPUD templates suspended in water with those obtained from SPUD templates spiked into sample preparations. The example in Fig. 4a shows the huge range of $C_{\mathrm{q}}$ s obtained with samples extracted from 


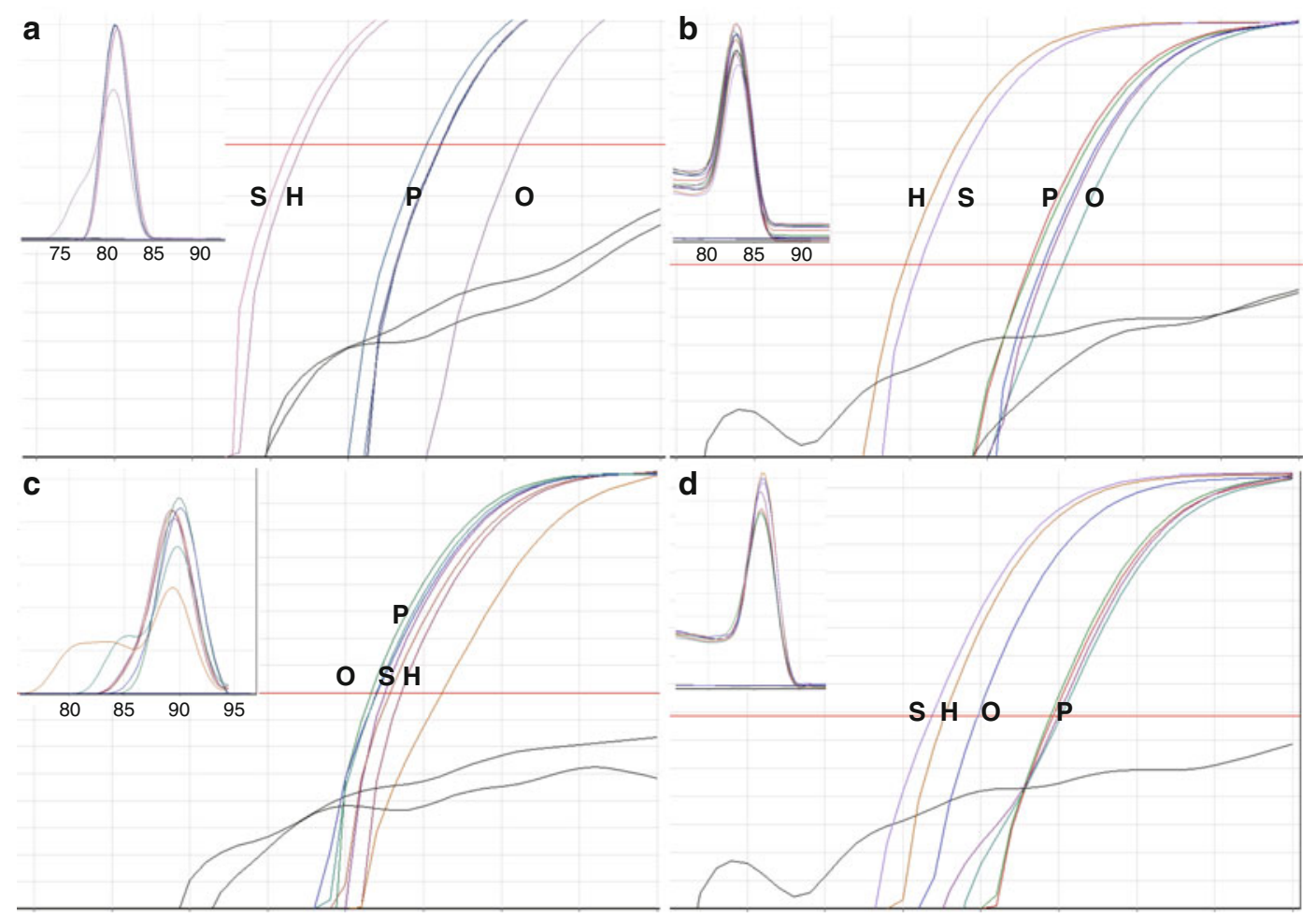

Fig. 2 Effect of priming strategy on final $C_{q}$. Equal amounts of RNA (RIN $\left.=10\right)$ were reverse transcribed using gene-specific primers $(\mathrm{S})$, random hexamers $(\mathrm{H})$, oligo-dT $(0)$, or pentadecamers $(\mathrm{P})$ at different concentrations, (a) DRG-1 (b) p21 (c) E-cadherin (d) 0steopontin. The inserts show the respective melt curves. All assays were carried out on a Corbett $6000 \mathrm{qPCR}$ instrument $\left(95^{\circ} \mathrm{C}, 10 \mathrm{~s} ; 59^{\circ} \mathrm{C}\right.$ or $\left.60^{\circ} \mathrm{C}, 15 \mathrm{~s} ; 72^{\circ} \mathrm{C}, 30 \mathrm{~s}\right) \times 40$

\section{Table 1}

\section{Details of primers and amplicons}

\begin{tabular}{|c|c|c|c|c|c|c|}
\hline Accession no. & Name & Primers & $\begin{array}{l}\text { Ta } \\
\left({ }^{\circ} \mathrm{C}\right)\end{array}$ & $\begin{array}{l}\text { Efficiency } \\
\text { (\%) }\end{array}$ & $\begin{array}{l}\text { Amplicon } \\
\text { size (bp) }\end{array}$ & $\begin{array}{l}\text { Position } \\
\text { (start) }\end{array}$ \\
\hline NM_006096 & DRG-1 & $\begin{array}{l}\text { CGATTTGCTCTAAACAACCCTGAG } \\
\text { CATCCAGCCTTCCGCACAAG }\end{array}$ & 58 & 100 & 78 & 582 \\
\hline NM_000582 & Osteopontin & $\begin{array}{l}\text { TTAAACAGGCTGATTCTGGAAGTTC } \\
\text { GATTCTGCTTCTGAGATGGGTCA }\end{array}$ & 60 & 99 & 105 & 221 \\
\hline NM_000389 & $\mathrm{p} 21$ & $\begin{array}{l}\text { CTGGAGACTCTCAGGGTCGAA } \\
\text { GGATTAGGGCTTCCTCTTGGA }\end{array}$ & 60 & 99 & 98 & 523 \\
\hline NM_004360 & E-cadherin & $\begin{array}{l}\text { TCCTCAGAGTCAGACAAAGACCAG } \\
\text { TCCTCGCCGCCTCCGTAC }\end{array}$ & 59 & 100 & 95 & 2,672 \\
\hline NM_001168 & Survivin & $\begin{array}{l}\text { CAGTGTTTCTTCTGCTTCAAGGAG } \\
\text { AGCGCAACCGGACGAATG }\end{array}$ & 62 & 98 & 90 & 287 \\
\hline NM_002467 & $\mathrm{c}-\mathrm{myc}$ & $\begin{array}{l}\text { TGAGGAGACACCGCCCAC } \\
\text { CAACATCGATTTCTTCCTCATCTTC }\end{array}$ & 62 & 100 & 71 & 1,292 \\
\hline
\end{tabular}



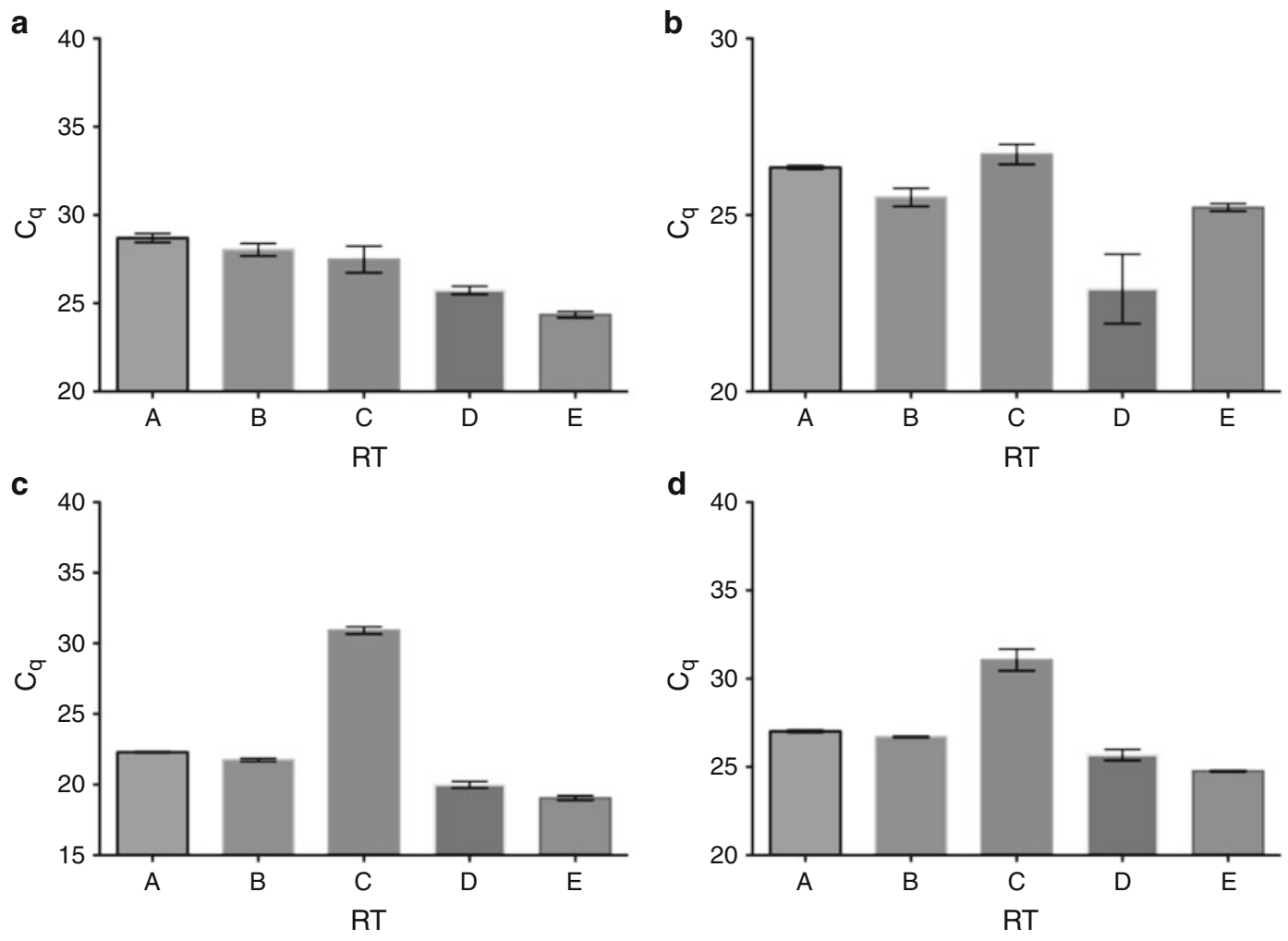

Fig. 3 Different properties of five different RTs. (a) p53; (b) c-myc; (c) GAPDH; (d) survivin
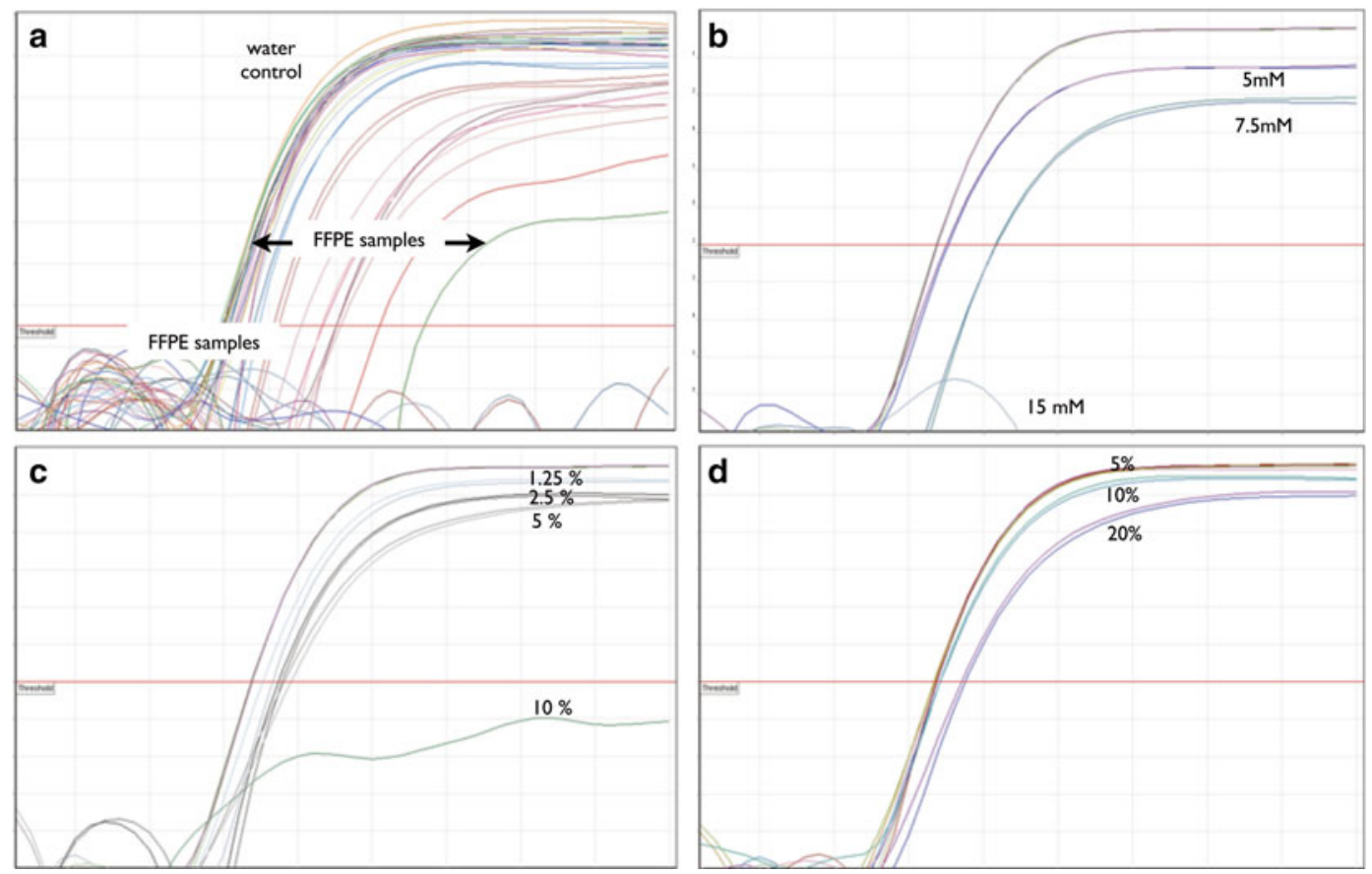

Fig. 4 Inhibition of qPCR assays using SPUD as a reporter. (a) FFPE extracted RNA samples; (b) EDTA; (c) Phenol; (d) Ethanol 
formalin fixed, paraffin-embedded samples, indicative of significant inhibition of most samples. Figure $4 \mathrm{~b}-\mathrm{d}$ shows the effects of three different common inhibitors of the PCR reaction on the SPUD assay. An interesting, and so far unexplained observation is that the slopes of the reactions remain rather similar, whereas the $C_{\mathrm{q}} \mathrm{s}$ increase with increasing concentrations of inhibitor. Whilst the SPUD assay is useful for general detection of inhibition, it has become clear that inhibition is not a simple process that affects each and every template to the same degree. Rather, testing for inhibition of individual targets within a sample is important, as we have demonstrated that inhibitors affect different PCR reactions to different extents [17].

The question of how to normalize appropriately when measuring RNA levels is one that has been around since the early days of RT-qPCR [18] and continues to be dealt with in a wholly unsatisfactory manner [19]. The problem is that in addition to problems associated with reverse transcriptases, priming methods, inhibitors, and PCR efficiencies there is an inherent variability, i.e., error associated with RNA itself and with the protocols used for its extraction. This requires the application of a consistent, appropriate, and accurate method of normalization to control for that error. There are several normalization strategies, none of which are mutually exclusive and all of which can be incorporated into a protocol at many stages [20]. For now, the use of reference genes represents the strategy that is most widely accepted, but they must be validated within the context of each individual experimental setup if the data are to be biologically meaningful $[21,22]$. As a general rule, if RNA levels differ by $>50$-fold, there is no real need for a reference gene and normalization against total RNA is sufficient. If target levels differ by between six- and tenfold, a single reference gene may suffice, especially if comparisons are carried out between single cell lines. If two samples differ by $<5$-fold in their RNA levels, it is essential to use multiple reference genes, an approach that is robust and allows accurate normalization if fine measurements are to be made. Even then, the resolution of the particular assay remains dependent on the sample and variability of the chosen reference genes. For example, it will be far more difficult to find a set of reference genes that vary by $<3$-fold when using colorectal cancer samples from individual patients than if using colorectal cancer cell lines.

\section{Considerations for the Future}

The breakthrough of MIQE and its acceptance by the wider research community is welcome and the increasing inclusion of the various quality control parameters will undoubtedly result in the publication of peer-reviewed publications of a higher technical standard. It is unfortunate that it has taken such a long time to 
draft, publish, and disseminate these guidelines, as the peerreviewed scientific literature now comprises thousands of publications that have erroneous conclusions based on inappropriate qPCR results. Anyone who has ever looked for publications supporting one or the other of two opposite viewpoints is very likely to find a number of publications supporting either position [23]. This is vexing and very likely will continue to lead to intellectual diversion as well as further investment in wasted cost and time.

However, another specter is even more thought-provoking and constitutes a logical extension of previously published information. The excellent investigations into the properties of RTs discussed above $[14,15]$ concluded that the type of RT, the priming strategy, and the amount of RNA used can generate significantly different results. It has been argued that this does not affect the ability to obtain comparable RT-qPCR, since the RT reaction is highly reproducible as long as the same experimental protocol and reaction conditions are used [24]. However, the RT step is not necessarily linear across different targets, with significant differences between the detection limits of different RTs that may be due to some components in the RT system that bias the subsequent PCR amplification [25]. Hence this viewpoint misses the inescapable truth that if four laboratories use four different RTs, four different priming strategies (gene-specific primers, hexamers, pentadecamers, oligo-dT), and different experimental protocols (widely different amounts of RNA, different volumes, different temperatures and times), they can end up with significantly different results despite following best practice protocols. The fact that each of these groups complies with the MIQE guidelines when publishing their data helps understand why the results may be different, but does not indicate which of the results is likely to be the correct one. These same authors concede, that we have no idea how the isolation yield varies among different mRNAs as differences in length, folding, localization in the cell, and complex formation with proteins are just some factors that may affect RNA extraction yield. This adds another level of error, and again it is perfectly conceivable that all extraction protocols are performed to the highest possible standards, that the RNA is quality assessed and handled appropriately, but that the additional variability introduced by the variable protocols will add to the RT-based errors, making any meaningful comparison of data very difficult. If this is then extended to the evaluation of reference genes, it is not inconceivable that different research groups will end up with different reference genes, and that the normalization of the RT-qPCR data will introduce yet one more level of variability. Importantly, all the results are technically accurate and deliver results that are repeatable and may even be reproducible, but depend entirely on how the experiments were performed. This is a real Achilles heel of the RT-qPCR technology, and is a problem that has not yet been addressed in a satisfactory manner. Perhaps the introduction of digital PCR will help somewhat reduce the distortion introduced by 
relative quantification, but by itself it does not tackle the problems of the RT step. Perhaps the solution is to focus our efforts on the quantification of proteins using PCR with proximity ligation or extension assays, and determine the levels of proteins, rather than the highly variable and, possibly, biologically less relevant levels of mRNA or even miRNAs. Proteins are, after all, the molecules that have function and identifying changes in the levels of proteins may be more informative than simply counting mRNA levels. Of course, protein-targeting introduces a whole new range of problems, but this should not distract from posing the question whether the only point of quantifying mRNA or miRNA levels by qPCR is to investigate a narrow set of regulatory mechanism, but that this approach is unlikely to yield information on the wider question of how and why cells alter their behavior in response to stimuli, or why normal cells develop into cancer cells. Changes to RNA transcript levels are, after all, a very small part of the overall mechanism of gene expression that involves huge numbers of proteins and their isoforms with different extents of posttranslational modifications, some cleaved and others complexed into active forms and all directly relevant to cell behavior (Fig. 5). On the other hand, many RNAs are useful as

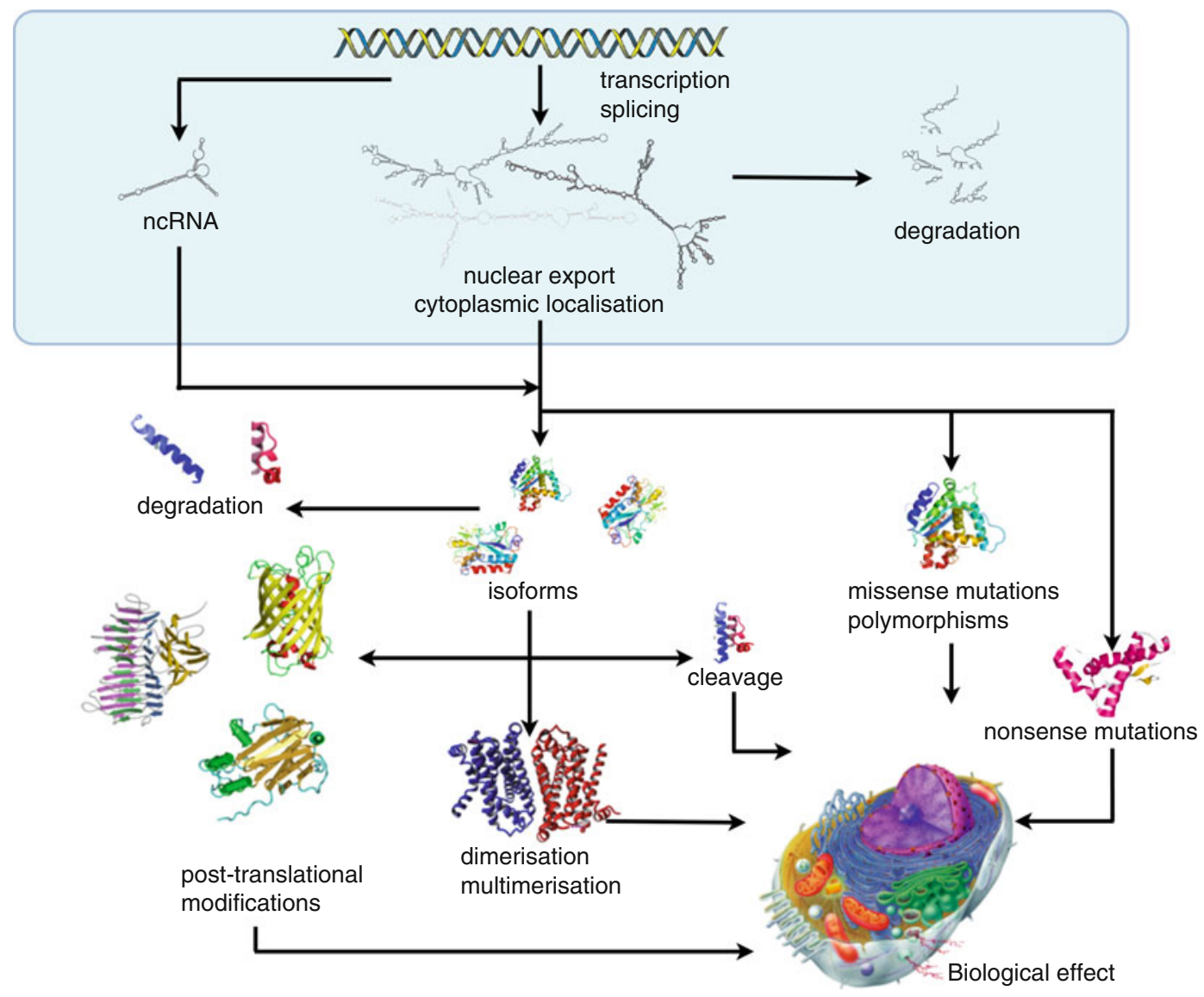

Fig. 5 The complexity of the gene expression pathway 
diagnostic or prognostic biomarkers, in which case the use of RT-qPCR will continue to be necessary and valuable, but will have to be carried out with regard to the problems outlined above.

In conclusion, biologically relevant qPCR results depend on many parameters that must all come together in an appropriate, controlled, and transparent manner. These are highlighted by the MIQE guidelines, which are increasingly seen as a valuable sentinel safeguarding the reliability and reproducibility of qPCR-based conclusions. The urgent need for their implementation is vividly demonstrated by a recent publication that reviewed over 2,000 publications for compliance with the MIQE guidelines and found that the vast majority report inadequate or inappropriate information [26]. MIQE aims to make nucleic acid analysis not just easy but reliable and allow qPCR to exploit its wide range of applications that today range from the quantification of RNA to epigenetics and protein detection, using variations on essentially the same theme. Addressing one set of challenges opens up new ones, and as applications of this simple technique continue to diversify, there will be a greater need for more bespoke considerations.

\section{References}

1. Bustin SA, Benes V, Garson JA et al (2009) The MIQE guidelines: minimum information for publication of quantitative real-time PCR experiments. Clin Chem 55:611-622

2. Bustin SA (2013) Why there is no link between measles virus and autism. In: Fitzgerald $M(e d)$ Recent advances in autism spectrum disorders, vol I, Intech. Rijeka, Croatia, pp 81-98

3. Kakisi OK, Robinson MJ, Tettmar KI et al (2013) The rise and fall of XMRV. Transfus Med 23:142-151

4. Roehr B (2012) Researchers find no link between XMRV and chronic fatigue syndrome. BMJ 345:e6331

5. Bohlenius H, Eriksson S, Parcy F et al (2007) Retraction. Science 316:367

6. Bustin SA (2010) Why the need for qPCR publication guidelines?-The case for MIQE. Methods 50:217-226

7. Abdel Nour AM, Pfaffl M (2011) Apple store, apple https://itunes.apple.com/app/miqeqpcr/ id423650002? $\mathrm{mt}=8$ or https://play.google. com/store/apps/details? id=com. biorad . miqeqPCR

8. Anonymous (2013) Announcement: reducing our irreproducibility. Nature 496:398

9. Shaffer C (2010) Routine lab method's accuracy called into question. Nat Med 16:349

10. Marx V (2013) PCR: living life amplified and standardized. Nat Methods 10:391-395
11. Bustin SA, Beaulieu JF, Huggett J et al (2010) MIQE precis: practical implementation of minimum standard guidelines for fluorescencebased quantitative real-time PCR experiments. BMC Mol Biol 11:74

12. Huggett JF, Foy CA, Benes V et al (2013) The digital MIQE guidelines: minimum information for publication of quantitative digital PCR experiments. Clin Chem 59:892-902

13. Bustin SA, Benes V, Garson JA et al (2011) Primer sequence disclosure: a clarification of the MIQE guidelines. Clin Chem 57:919-921

14. Stahlberg A, Hakansson J, Xian X et al (2004) Properties of the reverse transcription reaction in mRNA quantification. Clin Chem 50: 509-515

15. Stahlberg A, Kubista M, Pfaffl M (2004) Comparison of reverse transcriptases in gene expression analysis. Clin Chem 50: $1678-1680$

16. Nolan T, Hands RE, Ogunkolade BW et al (2006) SPUD: a qPCR assay for the detection of inhibitors in nucleic acid preparations. Anal Biochem 351:308-310

17. Huggett JF, Novak T, Garson JA et al (2008) Differential susceptibility of PCR reactions to inhibitors: an important and unrecognised phenomenon. BMC Res Notes 1:70

18. Bustin SA (2000) Absolute quantification of mRNA using real-time reverse transcription polymerase chain reaction assays. J Mol Endocrinol 25:169-193 
19. Huggett J, Bustin SA (2011) Standardisation and reporting for nucleic acid quantification. Accredit Qual Assur 16:399-405

20. Huggett J, Dheda K, Bustin S et al (2005) Real-time RT-PCR normalisation; strategies and considerations. Genes Immun 6:279-284

21. Tricarico C, Pinzani P, Bianchi S et al (2002) Quantitative real-time reverse transcription polymerase chain reaction: normalization to rRNA or single housekeeping genes is inappropriate for human tissue biopsies. Anal Biochem 309:293-300

22. Dheda K, Huggett JF, Chang JS et al (2005) The implications of using an inappropriate reference gene for real-time reverse transcription PCR data normalization. Anal Biochem 344: 141-143
23. Bustin SA, Mueller R (2005) Real-time reverse transcription PCR (qRT-PCR) and its potential use in clinical diagnosis. Clin Sci (Lond) 109: 365-379

24. Kubista M, Andrade JM, Bengtsson $M$ et al (2006) The real-time polymerase chain reaction. Mol Aspects Med 27:95-125

25. Levesque-Sergerie JP, Duquette M, Thibault C et al (2007) Detection limits of several commercial reverse transcriptase enzymes: impact on the low- and high-abundance transcript levels assessed by quantitative RT-PCR. BMC Mol Biol 8:93

26. Bustin SA, Benes V, Garson J et al (2013) The need for transparency and good practices in the qPCR literature. Nat Methods 10: 1063-1067 